\title{
Criminologie
}

\section{Prévenir le vol à main armée ?}

\section{Donate Poirier}

Volume 18, numéro 2, 1985

\section{Le vol à main armée à Montréal}

URI : https://id.erudit.org/iderudit/017220ar

DOI : https://doi.org/10.7202/017220ar

Aller au sommaire du numéro

\section{Éditeur(s)}

Les Presses de l'Université de Montréal

\section{ISSN}

0316-0041 (imprimé)

1492-1367 (numérique)

Découvrir la revue

\section{Citer cet article}

Poirier, D. (1985). Prévenir le vol à main armée ? Criminologie, 18(2), 135-145. https://doi.org/10.7202/017220ar

\section{Résumé de l'article}

Armed robbery seems to be on the decline in Montreal. But there, as everywhere, prevention is "in».

Are there effective ways to prevent armed robbery? To answer this question, the study compared the various means used by a sample of 271 Montreal shop owners : 184 of them had been victims of robbery during the last two years and $87 \mathrm{had}$ as yet never been robbed.

There are no easy solutions apart from selling the business. It was found that almost all retailers were prevention conscious. Non-expensive equipment is used in most stores by victims as well as non-victims. Costly means, such as alarms or cameras, are not very common but their preventive effect, if any, could not be other than indirect.

Cautious behavior, available to all, seems more effective. Non-victims had adopted slightly more preventive habits than former victims, such as frequent and irregular bank deposits, and/or enhancing the shop's visibility, etc. But so many more factors contribute to crime, several of which are beyond the control of the victim. Prevention also has negative side-effects. Is it worth it?
Ce document est protégé par la loi sur le droit d'auteur. L'utilisation des services d’Érudit (y compris la reproduction) est assujettie à sa politique d'utilisation que vous pouvez consulter en ligne.

https://apropos.erudit.org/fr/usagers/politique-dutilisation/ 
Armed robbery seems to be on the decline in Montreal. But there, as everywhere, prevention is «in».

Are there effective ways to prevent armed robbery? To answer this question, the study compared the various means used by a sample of 271 Montreal shop owners : 184 of them had been victims of robbery during the last two years and 87 had as yet never been robbed.

There are no easy solutions apart from selling the business. It was found that almost all retailers were prevention conscious. Non-expensive equipment is used in most stores by victims as well as non-victims. Costly means, such as alarms or cameras, are not very common but their preventive effect, if any, could not be other than indirect.

Cautious behavior, available to all, seems more effective. Non-victims had adopted slightly more preventive habits than former victims, such as frequent and irregular bank deposits, and/or enhancing the shop's visibility, etc. But so many more factors contribute to crime, several of which are beyond the control of the victim. Prevention also has negative side-effects. Is it worth it?

\section{INTRODUCTION}

Depuis quelque temps, l'accent en matière de prévention a été mis sur l'environnement physique et ses relations avec les phénomènes de victimisation. Cette thèse souligne le rôle, parfois incitateur, de certains individus à devenir des victimes : accès facile, mauvais éclairage, isolement, pour ne citer que quelques exemples, augmentent leur vulnérabilité. L'innovation dans le domaine de la prévention se situe aux frontières de la théorie et de la pratique. Dorénavant, on s'attaque aux structures physiques de l'environnement tout en apportant une attention particulière à la création d'une meilleure cohésion sociale. Les points névralgiques de l'intervention deviennent donc multiples : l'offenseur, la victime, l'envi-

* L'auteur tient à remercier Micheline Baril, Ph.D., ainsi que Anne Morissette, M.Sc. (Crim.) et Pierre Pinsonneault, M.Sc. (Pol.), M.Sc. (Crim.) pour leur précieuse collaboration.

** Assistante de recherche, Centre international de criminologie comparée, Université de Montréal. 
ronnement social et les lieux physiques. Ces éléments, d'ailleurs ont tous un effet d'entraînement dans la lutte contre la criminalité. Au Québec, des projets de prévention tels que TANDEM, Opération commerce sont des applications de ces théories.

Malgré une évolution en ce qui a trait au développement du concept de prévention dans le temps, une difficulté d'ordre épistémologique persiste encore : comment évaluer son efficacité réelle? Ce problème découle directement de notre ignorance des causes véritables et précises de la criminalité. Et on se retrouve face à ce vieux dilemme des sciences humaines : l'absence d'événements criminels dans un milieu donné est-il dû à l'utilisation de mesures préventives, ou à autre chose?

Si chez les penseurs et chercheurs, les opinions varient en ce qui concerne le domaine de la prévention de la criminalité, certaines données statistiques nous permettent de croire qu'il en est de même pour les principaux intéressés : les éventuelles victimes. En effet, dans une étude récente dirigée par Micheline Baril (1984) à l'École de criminologie de l'Université de Montréal, 440 commerçants montréalais, propriétaires, employés, victimes ou non victimes de vol à main armée ont répondu à un questionnaire portant, entre autres choses, sur la prévention des vols à main armée dans les petits commerces.

Dans un volet de cette recherche plus globale sur les victimes de vol à main armée, nous nous sommes particulièrement intéressés à la prévention, en établissant une comparaison entre les victimes et les non-victimes. Nous livrons quelques-unes de nos constatations dans le présent article ${ }^{t}$.

Il ressort de cette étude que des commerçants propriétaires sont fort peu convaincus de la possibilité d'éliminer totalement ou de façon importante ce fléau auquel leur occupation les expose. En effet, plus de $80 \%$ d'entre eux considèrent que «quoique l'on fasse, il y aura toujours des vols à main armée». Interrogés cependant sur celui à qui incomberait la responsabilité de la prévention dans ce domaine, environ les trois quarts des commerçants ont exprimé l'opinion que «si les gens prenaient plus de précautions, il y aurait moins de hold up».

1. La méthodologie de cette recherche est succinctement décrite dans l'article de Baril, M., et Morissette, A., qui précède. 
Dans la même foulée, plus de la moitié des personnes interrogées dans ce sondage allèguent que «c'est à la police à faire de la prévention». Et, d'autre part, elles affirment dans une large mesure, c'est-à-dire pour plus de $75 \%$ d'entre elles, qu'elles sont en total désaccord avec le principe «qu'il faut se faire justice soimême et se défendre par la force». Et dans ce sens, plus de la moitié de ce même échantillon entérine la proposition "que le gouvernement augmente leurs impôts pour développer des pro. grammes de prévention», ce qui dénote leurs préoccupations face au problème entre autres du vol à main armée dans les commerces.

Nous allons, dans les pages suivantes, examiner les précautions prises dans ces petits commerces face au danger que représente les vols à main armée. Elles sont de deux ordres, il y a d'abord les moyens techniques utilisés par des commerçants pour prévenir le vol à main armée et d'autre part les habitudes préventives qu'ils adoptent.

\section{A. LES MOYENS DE PRÉVENIR LE VOL À MAIN ARMÉE}

Plusieurs moyens, plus ou moins dispendieux financièrement pour les petits commerçants, peuvent être utilisés pour restreindre les vols à main armée ou à tout le moins en limiter les dégâts. Parmi ceux-ci, soulignons des moyens de détecter l'éventuel voleur tels que les systèmes d'alarme, des portes ou vitrines spéciales où encore des caméras permettant de le protographier. L'utilisation du coffre-fort, de l'intercaisse, d'une minuterie, d'autre part, offrent l'avantage de soustraire de lourdes sommes de la caisse enregistreuse. Enfin, des moyens plus radicaux tels que l'embauche d'un garde de sécurité ou encore l'acquisition d'un chien de garde constituent des éléments de protection fort dissuasifs pour les voleurs mais également fort onéreux pour les commerçants. Le tableau suivant nous donne un aperçu de l'utilisation effective chez les propriétaires victimes ou non victimes des différents moyens que nous venons d'énumérer.

\section{a) Les moyens de détection}

Il ressort de ce tableau que l'achat d'un système d'alarme demeure l'instrument de prévention que prévilégie la majorité des propriétaires, puisque $60 \%$ d'entre eux nous ont déclaré qu'ils en possédaient. Cependant, certaines réserves doivent être apportées quant à son efficacité dans le contexte d'une tentative de vol 
TABLEAU 1

Moyens de prevention

\begin{tabular}{lccc}
\hline & Victimes $\%$ & Non-victimes \% & Tous \% \\
\hline Système d'alarme & 60,9 & 59,8 & 60,3 \\
Coffre-fort & 33,2 & 21,9 & 25,9 \\
Intercaisse & 21,2 & 21,8 & 21,4 \\
Minuterie & 17,4 & 11,5 & 15,5 \\
Portes, vitrines spéciales & 16,9 & 27,6 & 20,3 \\
Cameras & 9,2 & 4,6 & 7,3 \\
Chien de garde & 2,7 & 6,9 & 4,1 \\
Garde de sécurite & 2,2 & 1,2 & 1,9 \\
\hline
\end{tabular}

Victimes : 184

Non-Victimes : 87

$\mathrm{N}:: 271$

à main armée. Surtout utilisé pour contrer les vols par effraction, l'expérience démontre que son utilisation est restreinte, aléatoire, voire même dangereuse lors d'un vol à main armée. Les victimes. dans le déroulement de l'action oublient quelquefois d'actionner le mécanisme d'alarme et, d'autre part, le fait d'y avoir recours lors d'un vol à main armée peut déclencher une réaction agressive chez les voleurs, ce qui contribuerait à augmenter les risques de blessures, voire même de pertes de vie. D'ailleurs, pour plusieurs propriétaires, la conservation de la vie demeure primordiale puisque parmi ceux qui conseillent leurs employés sur les attitudes à adopter lors d'un vol à main armée, $85 \%$ d'entre eux estiment qu'il vaut mieux «donner l'argent» et "obéir» aux demandes des voleurs, plutôt que de résister. En somme, si la prudence est de mise, le seul fait de déclencher une sonnerie d'alarme, au vu et au su de l'infracteur peut contribuer à augmenter le taux de danger.

Quoique l'emploi de portes ou vitrines spéciales suscite un intérêt moins marqué chez l'ensemble de nos répondants $(20 \%$ de l'ensemble), on remarque cependant qu'il est plus accentué chez les non-victimes. Des arguments nombreux et diversifiés peuvent expliquer la faible popularité de ce moyen de prévention. Ainsi, par exemple, l'utilisation de portes barrées et contrôlées de l'intérieur du commerce peut amener quelques clients à modifier leur choix de lieu d'achat, ce qui contribue donc à faire supporter des 
pertes financières aux commerçants. De plus, la décision de laisser pénétrer un éventuel client demeure très arbitraire, elle ne s'appuie que sur le jugement de l'instant, sur des impressions générales. En effet, entre un client de passage, un nouveau locataire dans le quartier ou un voleur potentiel, comment distinguer? Également les coûts qu'entraînent ces dispositifs ne sont pas à sous-estimer pour le budget d'un petit commerçant, c'est pourquoi certains préfèrent souvent investir dans d'autres sphères de la prévention.

L'utilisation d'une caméra qui permet la prise de photographies lors d'un vol à main armée est également une pratique fort peu répandue en général, puisqu'à peine $8 \%$ de notre échantillon y a recours, les victimes, assez ironiquement, y font appel plus souvent que les non-victimes. Le coût de ce système peut être minime, car il existe sur le marché de fausses caméras mais qui donnent l'impression au voleur qu'il est photographié, cependant, même les vraies caméras sont peu dissuasives car il suffit de constater la piètre qualité de la photo.

Bien que les moyens que nous venons d'énumérer n'ont qu'un effet indirect sur la perpétration d'un vol à main armée, leur présence dans un commerce devrait, semble-t-il, contribuer à dissuader le voleur de passer à l'acte. L'hypothèse qui veut que le voleur devant ces dispositifs soit amené à changer de cible est une croyance partagée par certains criminologues et largement diffusée par les vendeurs de gadgets sécuritaires. Or, il ressort de notre analyse que si nos deux groupes (victimes et non-victimes) font appel en proportion égale à des systèmes d'alarme, c'est chez les non-victimes que l'on retrouve le plus souvent les systèmes de portes et vitrines spéciales, alors que c'est chez les victimes, en proportion modeste cependant, que l'on retrouve le plus souvent des caméras.

\section{b) Les moyens pour soustraire de l'argent de la caisse enregistreuse}

Basé sur le principe qu'il faut dégarnir le plus possible le contenu du tiroir de la caisse enregistreuse, pour d'une part minimiser les pertes monétaires en cas de vol et, d'autre part, rendre le commerce moins attrayant pour les observateurs avertis, le coffre-fort, l'intercaisse ou la minuterie, quoique fonctionnant différemment, ont tous la même fonction : conserver précieusement les recettes du commerce hors de la portée des voleurs. 
Considérées indépendamment, nos données révèlent que $26 \%$ des propriétaires privilégient l'emploi d'un coffre-fort plutôt que l'intercaisse qu'ils adoptent dans $21 \%$ des cas. La minuterie est le système de prévention le moins populaire, n'apportant les suffrages que de $15 \%$ des commerçants interviewés. Si pris individuellement, ces différents moyens n'ont qu'une faible teneur d'adhérants, nous constatons cependant que collectivement plus de $60 \%$ des propriétaires se sont munis d'un dispositif quelconque pour protéger leur argent, ce qui selon nous manifeste un souci présent et constant de préservation et de prévention dans ce domaine.

Face à ces données, nous pouvons nous demander si les commerçants, conscients de leur impuissance face au vol à maị armée ont tout simplement opté pour une attitude de conciliation réaliste et peut-être fataliste, c'est-à-dire dégarnir le plus possible leur caisse enregistreuse de sommes importantes pour ainsi amoindrir le bilan des pertes. Notre sondage qui nous a en effet démontré que huit commerçants sur dix considèrent que «depuis cinq ans les vols à main armée ont augmenté dans les commerces», explique peut-être en partie le fait que c'est chez les victimes que l'on retrouve le plus d'instruments (coffre-fort, et minuterie) pour amoindrir les pertes financières dues au vol, l'intercaisse jouissant d'une faveur égale de part et d'autre.

\section{c) Les moyens de protection}

L'emploi d'un garde de sécurité ou l'acquisition d'un chien de garde constituent des moyens directs et radicaux pour prévenir un vol à main armée. Présents sur la scène du délit, gardes ou chiens peuvent intervenir sur le champ et souvent leur seule présence a un effet dissuasif. Dans les faits, cependant, peu de propriétaires ont recours à cette ressource. En effet, le tableau 1 nous révèle que dans l'ensemble seulement $4 \%$ d'entre eux possèdent un chien d'attaque (avec une légère supériorité pour les non-victimes) et seulement $2 \%$ utilisent les services de garde de sécurité.

Des raisons financières peuvent expliquer ces faibles taux. En effet, pour beaucoup de petits commerçants il s'agit souvent d'investir des frais considérables, compte tenu d'un chiffre d'affaires modeste. Un garde de sécurité, même payé au salaire minimum, leur est à peu près inaccessible et combien coûte le meilleur ami du commerçant? 
Premièrement, ça coûte huit cents piastres un chien, et vous avez la loi, la vraie loi qu'on n'a pas le droit d'avoir un chien dans un établissement commercial, c'est ben défendu question d'hygiène, pis ça peut être dangereux pour les enfants pis les autres, parce qu'un chien écoute seulement une personne, il est dressé pour ça, y peut blesser quelqu'un, j'ai pas osé moi... (Manseau, 1977, p. 165).

En somme, au niveau des moyens préventifs, on constate que si victimes et non-victimes font également appel à des systèmes d'alarme et d'intercaisse, les victimes font, plus souvent que les autres, confiance au coffre-fort, à la minuterie et au système de caméra. Les non-victimes se particularisent par un emploi plus large de portes et de vitrines spéciales.

\section{B. LES HABITUDES PRÉVENTIVES}

Contrairement à ce qui précède, certaines habitudes en matière de prévention n'entraînent aucun déboursé direct. Reliées au quotidien des commerçants, elles démontrent leurs attitudes dans leur démarche personnelle vis-à-vis de la prévention. Variées, ces habitudes peuvent s'appliquer aux structures du commerce tel que, par exemple, le fait d'éviter de tapisser les vitrines d'affiches publicitaires et le fait d'avoir un éclairage adéquat à l'intérieur du commerce et ceci afin de favoriser une meilleure vision de ce qui s'y passe, autant de l'extérieur que de l'intérieur. Également, le commerçant peut, s'il le désire, prendre un certain nombre de précautions dans la manipulation des montants d'argent à l'intérieur comme à l'extérieur de son commerce. Ainsi, garder le minimum dans la caisse, exécuter des dépôts fréquents et changer sa routine dans cette politique de dépôts constituent des habitudes de prudence élémentaire, accessibles à tous. Dans le tableau suivant, nous présentons un éventail des différentes attitudes sécuritaires que peuvent adopter les commerçants ainsi que leurs fréquences d'application.

\section{a) Les habitudes relatives aux structures du commerce}

Dissuader le voleur potentiel de passer à l'acte fait partie de la stratégie préventive que peut, en tout premier lieu, amorcer un commerçant. Ainsi, en favorisant une meilleure visibilité de l'extérieur du commerce, on se procure l'avantage de profiter d'une certaine surveillance informelle de la part des passants dans 
TABLEAU 2

Les habitudes préventives

\begin{tabular}{lccc}
\hline & Victimes \% & Non-victimes \% & Tous \% \\
\hline Minimum dans la caisse & 72,8 & 96,6 & 80,4 \\
Bon éclairage & 73,4 & 90,8 & 79 \\
Dépôts fréquents & 60,3 & 79,3 & 66,4 \\
Changement de routine & 57,1 & 77 & 63,5 \\
Vitrines dégagées & 50,5 & 66,7 & 55,7 \\
\hline
\end{tabular}

Victimes : 184

Non-victimes : 87

N : 271

la rue. Dans ce sens, si plus des trois quarts de l'ensemble des propriétaires que nous avons rencontrés nous ont affirmé qu'ils considèrent que leur commerce était doté d'un *bon éclairage», tandis que $55 \%$ d'entre eux se souciaient de «dégager les vitrines», une constatation intéressante s'impose quand on compare les victimes et les non-victimes : en effet, c'est chez ces dernières que l'on fait le plus souvent appel à ces mesures élémentaires de prévention.

\section{b) Les habitudes relatives à la manipulation des sommes}

À cet effet, les résultats sont forts révélateurs. Conscients de l'importance de «garder le minimum d'argent dans la caisse» pour prévenir un éventuel vol ou à tout le moins en minimiser les dégâts, $80 \%$ des gens de notre échantillon pratiquent une telle habitude. Ici encore les non-victimes se distinguent des autres car elles ont adopté en quasi-totalité cette politique alors que les victimes, elles, n'y font appel que dans les trois quarts des cas. S'il n'y a que $66 \%$ de l'ensemble de nos répondants qui nous affirment effectuer des dépôts fréquents, et cela est probablement dû à la difficulté qu'ont certains commerçants de quitter leur établissement sur les heures d'ouverture - car plusieurs sont seuls à opérer leur commerce - les non-victimes le font, ici encore, beaucoup plus souvent que les autres, et ceci dans une proportion de six à huit. Enfin, si $63 \%$ de nos répondants changent de «routine» pour ainsi dérouter les éventuels observateurs qui planifieraient leur coup, les non-victimes, encore une fois le 
font plus fréquemment. En effet, il y a $20 \%$ de plus d'entres elles qui adoptent cette politique préventive, que de victimes. Faire des dépôts à toute heure du jour et de la nuit participe d'ailleurs d'une stratégie préventive, comme en fait foi le témoignage suivant :

On vide la caisse très régulièrement, maintenant. J'ai une caisse de dépôt et régulièrement, plus l'heure avance, il faut en mettre... il faut qu'il y ait le moins de circulation d'argent possible. A ce moment là, s'il y a un hold-up, pis qui reste $30 \$$ ou $40 \$$, généralement y sont pas intéressés à revenir (cité dans Grenier et Manseau, 1977, p. 69) .

Il y a donc, dans tous les cas, une plus grande proportion de propriétaires non victimes qui ont adopté des habitudes préventives, soit au niveau des structures de leur commerce (éclairage, vitrines), soit encore dans le domaine de la manipulation de l'argent, que ce n'est le cas pour leurs pairs victimes.

\section{CONCLUSION}

Ce qui ressort de notre étude, c'est un contraste important, entre les commerçants victimes de hold-up et ceux qui ne l'ont pas encore été, au niveau des habitudes de prévention, car à celui des moyens, bien qu'il existe un certain nombre de différences, elles sont quand même moins flagrantes.

En effet, au niveau des moyens, abstraction faite du fait que les deux groupes utilisent dans les mêmes proportions des systèmes d'alarme (véhicules plus ou moins efficaces en cas de hold-up comme nous l'avons vu) et qu'également ils se départagent à peu près également en faisant appel à des moyens pour soustraire des montants d'argent de leur caisse enregistreuse (que ce soit en faisant appel à un coffre-fort, un intercaisse ou un système de minuterie), si l'on fait exception également de moyens peu utilisés tels caméras, chiens de garde et gardes de sécurité : ce serait l'existence de portes et de vitrines spéciales chez les non-victimes qui les distinguent des commerçants victimes de hold-up.

Si des habitudes de prévention ne coûtent rien, les moyens sont quelquefois fort dispendieux pour des petits et moyens commerçants. Ceux qui ont été interrogés dans le cadre de notre recherche ont consacré en moyenne autour de $2000 \$$ pour en acquérir. À la limite, $30 \%$ d'entre eux investissant moins de $500 \$$, alors qu'une minorité de moins de $1 \%$ dépassait les $6000 \$$. 
Or, c'est surtout dans le domaine des habitudes préventives que le divorce entre victimes et non victimes est le plus profond. En effet, ces dernières adoptent et pratiquent dans une plus large proportion les conseils prodigués par les préventionnistes, policiers ou autres. Qu'il s'agisse de conserver un minimum d'argent dans la caisse, de faire des dépôts fréquents à la banque ou de modifier leurs habitudes dans ce dernier domaine, que ce soit encore au niveau de l'aménagement physique de leur local commercial, les non-victimes semblent mettre toutes les chances de leur côté pour éviter ou encore limiter les séquelles de ce contretemps fâcheux que constitue un hold-up.

Mais les commerçants, même très prudents réussiront-ils toujours à se barricader contre ce fléau que constitue le vol à main armée dans une grande agglomération urbaine comme Montréal? Il semble que non puisque, comme en font foi les résultats de notre recherche, même les commerçants victimes de hold-up ont eux aussi adopté, dans une moins large mesure, il est vrai, des moyens et des habitudes préventives.

Cette constatation peut nous amener à nous interroger sur l'efficacité réelle des politiques de prévention. D'après nos résultats, en effet, il semblerait que la prévention aurait une influence plutôt diffuse sur la perpétration ou non d'un vol à main armée. C'est que nous ne pouvons, par ailleurs, ignorer la présence d'autres facteurs difficilement mesurables au sein de notre présente recherche. Par exemple, la situation géographique du commerce, la familiarité avec les lieux, le nombre de personnes présentes dans le commerce, l'argent disponible, deviennent tous des composantes importantes dans le choix de la cible pour le voleur. De surcroît, comme le laissent entendre d'autres auteurs qui se sont attachés à la question :

Pour certains voleurs, le vol à main armée s'avère donc un moyen idéal de faire de l'argent rapidement. Il n'y a pas d'intermédiaire et l'argent est immédiatement disponible. De plus, la perpétration de l'acte proprement dit s'avère facile. Il demande relativement peu de planification et il s'exécute en quelques minutes (Normandeau, A. Pinsonneault, P., 1985, p. 12).

À la limite, le hasard dans le choix de la cible peut jouer un certain rôle. Comme le disait ce voleur :

... c'était un dimanche, $\mathrm{j}$ 'savais pas où aller pour chercher de l'argent. J'avais un gun mais pas de balles (...) j'ai vu le 
dépanneur. Un dimanche, t'as pas ben le choix. J'ai regardé le côté risque. J'avais besoin d'argent... (cité in Bellot et al., 1984, p. 55).

Relativement côteuse au niveau des moyens, souvent fastidieuse par les habitudes qu'elle impose aux commerçants, quelquefois désagréable par le traitement qu'elle fait subir à ses clients, plus ou moins efficace pour contrer un jeune voleur écervelé ou un bandit expérimenté et décidé, dangereuse même par les réactions qu'elle peut provoquer chez eux, la prévention somme toute, par les aspects négatifs qu'elle véhicule, en vaut-elle le coup?

\section{REFERENCES}

BEllot, S., M. DiONne, P. PINSONNEAUlt, M. CUSSON (1984), le Vol à main armée décrit par ses auteurs, Centre international de criminologie comparée, Université de Montréal, rapport final no 2, $153 \mathrm{p}$.

GRENIER, H., H. MANSEAU, M. BARIL (1977), les Petits commerçants victimes de vol à main armée, C.I.C.C.-G.R.A.C., Université de Montréal, rapport no 5, $185 \mathrm{p}$.

MORISSETTE, A. (1985), Subir un vol à main armée : réactions et conséquences, Centre international de criminologie comparée, Université de Montréal, rapport technique no $11,264 \mathrm{p}$.

NORMANDEAU, A., P. PINSONNEAULT (1985), le Vol à main armée à Montréal : les voleurs parlent, les victimes se prononcent..., Centre international de criminologie comparée, Université de Montréal, rapport final no 5, $187 \mathrm{p}$.

POIRIER, D., Prévention et vol à main armée, Centre international de criminologie comparée, Université de Montréal, rapport technique $\mathrm{n}^{\circ} 13$, (en cours). 